\title{
Anabases
}

ANABASES Traditions et réceptions de l'Antiquité

$20 \mid 2014$

Varia

\section{La malédiction de l'oreille. Pour Philippe Brunet}

André Markowicz

\section{OpenEdition}

Journals

Édition électronique

URL : http://journals.openedition.org/anabases/4888

DOI : 10.4000/anabases.4888

ISSN : 2256-9421

\section{Éditeur}

E.R.A.S.M.E.

\section{Édition imprimée}

Date de publication : 1 novembre 2014

Pagination : 79-84

ISSN : 1774-4296

\section{Référence électronique}

André Markowicz, «La malédiction de l'oreille. Pour Philippe Brunet », Anabases [En ligne], 20 | 2014

mis en ligne le 01 novembre 2017, consulté le 20 octobre 2019. URL : http://journals.openedition.org/ anabases/4888 ; DOI : 10.4000/anabases.4888 


\section{La malédiction de l'oreille. Pour Philippe Brunet}

André Markowicz

J'Ai Un BILINGUISME DÉFORMÉ. J'écris en français, j'entends en russe. Mais pas que le français : toutes les langues. Je me souviens comme j'ai été surpris, lisant le début de "L'Enfer ", quand un ami italien m'a dit que "Nel mezzo del camin di nostra vita ", ce n'était pas considéré comme un pentamètre iambique, alors que, si, évidemment, c'en est un. "Nel MEZzo del caMIN di NOStra Vita " (avec un accent sous-entendu sur l'article) ; j'avais dix-huit ans, et je ne comprenais pas que Dante n'ait pas vu une évidence pareille... J'ai dépassé la cinquantaine, je comprends que Dante avait d'autres soucis en tête, et, malgré tout, malgré moi, je me dis que, si, c'est un iambe, ce vers-là, et qu'il faudrait qu'on le lise comme ça.

C'est que le russe est syllabo-tonique. Et, mon oreille, elle est donc syllabo-tonique. Même en français. Plus on vieillit, plus on devient têtu.

Il y a chez Pouchkine un certain nombre de poèmes antiques. Antiques, au sens où Pouchkine cite des mètres antiques, et donc, fait une référence directe, par-delà le mètre, au genre (j'en ai cité un récemment, quand j'ai fini mes Ombres de Chine). Ces citations sont, la plupart du temps, des citations de l'amitié. Un ami de Pouchkine, Anton Delvig, écrivait en hexamètres russes, de vraies bucoliques, en distiques élégiaques russes, de vraies épigrammes anthologiques. - Il écrivait au moment où un autre poète, Nikolaï Gnéditch, traduisait l'Iliade, en hexamètres russes. En hexamètres russes, c'est-à-dire en hexamètres allemands écrits en russe.

Parce que ce sont les Allemands, je crois bien, qui, les premiers, ont adapté dans une langue moderne les mètres de l'Antiquité. Et qui l'ont fait d'une façon drastique : comme ils ne sentaient pas, dans leur langue, les accents d'intensité des 
langues antiques, ils les ont remplacés par ce qu'ils sentaient eux, c'est-à-dire les accents toniques, parce que ce dont il s'agissait, finalement, c'était juste de trouver un moyen pour faire entendre quelque chose de très ancien dans quelque chose d'encore à naître. Comme Lessing reprend le mètre de Shakespeare, le pentamètre iambique, pour créer le théâtre allemand (je vais vite, mais vous comprenez ce que je dis), de même, les poètes comme Klopstock, et, un peu plus tard, surtout, Goethe, recréent les mètres grecs, pour dire quelque chose d'indiscutable, c'est que l'Allemagne, ou, disons, la langue allemande, c'est la patrie de Shakespeare et d'Homère dans le monde moderne, et qu'elle est capable, cette langue, d'être la mémoire de l'Europe. - Je le dis en passant : imaginez Hölderlin sans la métrique antique... Lisez Brod und Wein sans lire le distique élégiaque... c'est juste absurde...

C'est donc par la métrique russo-allemande que je suis passé pour traduire les poèmes anthologiques de Pouchkine. En considérant que le français avait des accents toniques, même s'ils ne jouent pas de rôle dans la métrique traditionnelle, et s'ils sont fixes. - En fait, quand j'ai essayé, j'ai vu qu'un des poèmes que je voulais traduire l'avait déjà été, comme je pensais le faire, en faisant des distiques élégiaques français, par Jean-Louis Backès, qui faisait partie du groupe de traducteurs auquel j'appartenais, autour d'Efim Etkind. J'étais content, ça me prouvait que quelqu'un d'autre pouvait entendre le français comme moi, et qu'on n'avait pas besoin d'avoir grandi dans une autre langue pour ça.

Et puis, j’ai découvert Catulle. Par Ezra Pound - un ami, Armando Uribe Echeverria, qui lisait Pound, m'a montré un poème en trois vers :

\section{Siqui forte mearum ineptiarum \\ Lectores eritis manusque uestras \\ Non horrebitis admouere nobis..}

Littéralement : «Si jamais par hasard de mes sottises/Vous êtes lecteurs/ Et vous ne tremblez pas d'approcher vos mains de nous..."

Et, je ne sais pas pourquoi, je me suis dit que j'allais les traduire, ces trois vers. Armando disait : «André, ce qu'il aime, il veut le traduire ", et je pense que rarement on a dit quelque chose de plus juste sur moi.

Je connaissais déjà Catulle, en fait, mais pour un seul poème. Pour une traduction que Pouchkine avait faite du poème 27 « Minister uetuli puer Falerni »... Un petit poème de huit vers, que j'ai publié dans Le Soleil d'Alexandre: 
"L'âpre ivresse du Falerne

Vienne, enfant, emplir la coupe,

Puisque Postumia l'ordonne,

Qui préside à notre fête.

Vous, fuyez la joie bruyante,

Ondes ennemies des grappes,

Abreuvez le sage triste :

Nous aimons le pur Bacchus. »

En russe, c'est construit sur ce rythme saccadé ("trochaïque », ça s'appelle : unité de deux syllabes, accent sur la première), et sur les allitérations :

Пьяной горечью Фалерна

Чашу мне наполни, мальчик!

Так Постумия велела,

Председательница оргий.

Вы же, воды, прочь теките

И струёй, вину враждебной,

Строгих постников поите:

Чистый нам любезен Бахус

Le texte latin :

"Minister uetuli puer Falerni,

Inger me calices amariores,

Ut lex Postumiae iubet magistrae

Ebria acina ebriosioris.

At uos quo lubet hinc abite, lymphae,

Vini pernicies, et ad seueros

Migrate; hic merus est Thyionianus. "

Tout est génial dans ce poème - et surtout les allitérations que Pouchkine a su retranscrire (ce sont ces allitérations qui m’ont guidé, bien sûr, dans mon travail). Mais, pour ce poème, Pouchkine n'avait pas voulu traduire le mètre : le phalécien, parce qu'il n'avait aucun exemple de phalécien en russe, et que la citation du mètre n'aurait pas fait écho.

C'est quoi, un phalécien?

C'est ça :

$-1-\mathrm{u} u /-\mathrm{u} /-\mathrm{u}-\mathrm{u}$

MI NIS/TER uetu/LI pu/ER faLERni

Encore une fois, il ne s'agit pas, en latin, d'accents toniques, mais d'accents d'intensité : les syllabes sont longues ou brèves. Moi, j'entends des accents toniques. 
Et comment faire pour reproduire ces accents ? - Comment faire pour ne pas franciser l'étranger, pour nous rendre, une fois encore, étrangers, nous, dans notre propre langue ? Et même si ça ne correspond à rien chez nous... C'est l'idée de tout mon travail, ça, depuis le tout début, vraiment. Parce que, non, je ne peux pas faire autrement. Il faut que je suive ce que j'entends, partant de l'idée que, puisque je l'entends, d'une façon ou d'une autre, ça existe, ou ça peut exister...

Comme les Allemands, je pars de l'idée qu'une syllabe longue, c'est un accent tonique. Sauf que, ça n'existe pas, dans aucune langue européenne, du moins, trois accents toniques qui se suivent. L'idée est donc de décomposer, et de recomposer. Reprenons le schéma :

$$
-1-\mathrm{u} u /-\mathrm{u} /-\mathrm{u}-\mathrm{u}
$$

Un mètre, ce n'est pas un schéma. C'est un jeu vivant d'accents principaux et d'accents secondaires. Certains accents sont obligatoires -, sans quoi le mètre lui-même n'est pas reconnaissable. D'autres peuvent varier.

Quels seraient les accents principaux de ce vers?

Clairement, la position 1, ou la position 3, puis la position 6, puis la 8 et, naturellement, la 10 (le 11 étant aléatoire).

Ça veut donc dire que ça nous donne :

$-\mathrm{u}-/ \mathrm{u} \mathrm{u}-/ \mathrm{u}-/ \mathrm{u}-/ \mathrm{u}$

ou bien, plus facilement :

$\mathrm{u} \mathrm{u}-/ \mathrm{u} \mathrm{u}-/ \mathrm{u}-/ \mathrm{u}-/ \mathrm{u}$

parce qu'il est difficile, en français, de commencer par un accent tonique : il faudrait toujours commencer par un monosyllabe.

C'est-à-dire : anapeste + anapeste + iambe + iambe. C'est-à-dire que, métriquement, ça pourrait donner ça (dans une version re-re-re-refaite) :

"Jeune esclave qui sers un vieux Falerne,

Verse-moi une ivresse plus amère :

Postumia me l'ordonne, notre reine,

Ivre plus que la grappe la plus ivre.

Vous, dehors et bon vent, les ondes fades

Corruptrices du vin, fuyez chez l'homme

Grave. Ici Dionysos est sans mélange. »

J'ai travaillé pendant quatre ans à traduire tout Catulle. Je sais que c'était très très mauvais -, ces traductions (le livre est paru en 1985, c'était mon premier livre à moi tout seul...). Aujourd'hui, trente ans plus tard, j'ai ouvert mon édition sur le fragment dont j'ai parlé au début de ma chronique (dit fragment 14bis), et c'est tellement mauvais que je ne veux même pas le citer. - Serais-je capable, maintenant, d'en 
faire quelque chose ? Sans doute pas. Il y a, comme ça, des livres maudits, pour chaque auteur. Pourtant, en traduisant l'ensemble de son livre, j'ai trouvé en Catulle un des poètes qui me touchent le plus au monde. Et cet amour que j'éprouve pour lui, il ne s'est jamais démenti. Plus je le lis, plus il me semble prodigieux. Bien moins pour ses poèmes rageurs, obscènes, évidemment, que ses poèmes lyriques, ou, surtout, ses petites épopées - ses longs poèmes... Je tourne autour, depuis toujours, je me les récite, et je me dis que, oui, un jour, quand j'aurai le temps, si je pouvais refaire... une petite quarantaine de textes...

J'ai commencé à traduire Catulle en 1979, je crois bien. J'étais à la Sorbonne, avec Armando. Et, plus encore que lui, je haïssais la Sorbonne. Ce n'est pas que je la haïssais, à vrai dire, elle me glaçait. Les professeurs, qui me semblaient tous vieux (ils l'étaient), et, je ne sais pas, comme une espèce d'arrière-goût bizarre. J'ai appris par la suite que l'un de nos professeurs était membre du bureau politique du FN (à l'époque !...), un autre était membre d'un truc qui s'appelait "Ligue mondiale contre le bolchévisme »..., un autre était président de l'UNI, bref, je ne voulais pas les voir, ces gens-là. Et ce qu'ils " enseignaient »... Jésus-Marie... c'était tellement plat, tellement insignifiant, et tellement satisfait. Oui, c'était ça, surtout, qui me terrorisait : ils étaient contents de savoir, ces gens-là. Contents d'être les représentants éminents, ou les gardiens du temple d'une culture qui devait être la mienne, à moi aussi. Je ne voulais pas de ça.

Il y avait deux enseignants passionnants -, pas des profs : un jeune barbu qui faisait cours sur Homère, et qui s'appelait M. Durand (qu'est-il devenu ?), et une jeune femme qui faisait cours, elle, sur les latins : Florence Dupont... Bon, bref, tout ça pour dire que je n'allais pas à beaucoup de cours, et que je restais au café, pour travailler. J'y restais la journée entière. J'arrivais de ma banlieue tôt le matin, je me mettais au fond de la salle, et je travaillais. Je faisais salon aussi, en quelque sorte.

C'est comme ça, je crois bien, que, par Armando et une copine commune (laquelle est devenue depuis une très éminente et très brillante prof à la Sorbonne, Catherine Coquio), j'ai rencontré un garçon qui avait l'air japonais et qui faisait du hard rock. Japonais, il l'était par sa maman, et pas n'importe quel japonais, parce que, par sa maman, il était de la famille impériale... Il y a des gens, comme ça, qu'on rencontre, et dont on sait qu'ils sont très importants, tout de suite, dans votre vie. Philippe Brunet m’a fasciné par une chose : il n'affirmait jamais rien, il ne "savait " pas. Il écoutait, il avait une espèce de sourire timide et très sérieux. Et il se passionnait pour le grec (il n'avait pas fait de grec au lycée, il en faisait, là, en khâgne, à Louis le Grand). Il me parlait avec une grande tendresse d'un de ses professeurs, Pierre Fortassier, qui avait fait une étude sur les hexamètres spondaïques chez Virgile (oui, je crois, chez Virgile, pas 
chez Homère - il a écrit un livre sur Homère, pourtant), bref, je ne me souviens plus très bien, mais ce qui m'avait frappé, c'était ça : qu'on puisse se consacrer avec passion à un point si ténu, et voir dans ce point si ténu une clé qui permettait d'ouvrir une poétique. Il y en avait 17 -, peut-être que je me trompe ;- des hexamètres spondaïques, c'est-à-dire des hexamètres avec des spondées à la cinquième position, laquelle est la position décisive pour la reconnaissance du vers... Finalement, c'est de ça que je me souviens. De la passion pour un point de métrique... Et d'un seul coup, je me suis senti en confiance, moi, avec mon oreille étrangère et ma passion à moi. Ma passion russe pour le latin. Nous nous sommes sentis frères, je crois que je peux le dire.

Parce que, Philippe, il lisait l'allemand et il comprenait, lui, l'importance de la métrique antique pour lire Goethe, Schiller ou Hölderlin. Nous avions Hölderlin au programme... " Pauvre Pindare », comme on dit dans « La grande illusion »... Mais soudain, pas en parlant avec Philippe (parce que, finalement, que voulez-vous que nous nous disions ?), non, juste en étant avec Philippe, là, il y avait de la vie. Et puis, Philippe s'est passionné pour Pindare (je crois, par Hölderlin, parce qu'il comprenait bien que, le vers libre des dernières grandes odes, il venait de la contrainte extrême des Odes de Pindare). Et Philippe a tout de suite compris mon idée de remplacer les accents d'intensité par des accents toniques, et, avec son oreille musicale, il entendait... Et c'est comme ça, je crois, qu'a commencé, en souriant, envers et contre tout, une des aventures les plus extraordinaires de l'histoire de la traduction en France - la sienne.

André MARKOWICZ

a.marko@wanadoo.fr 